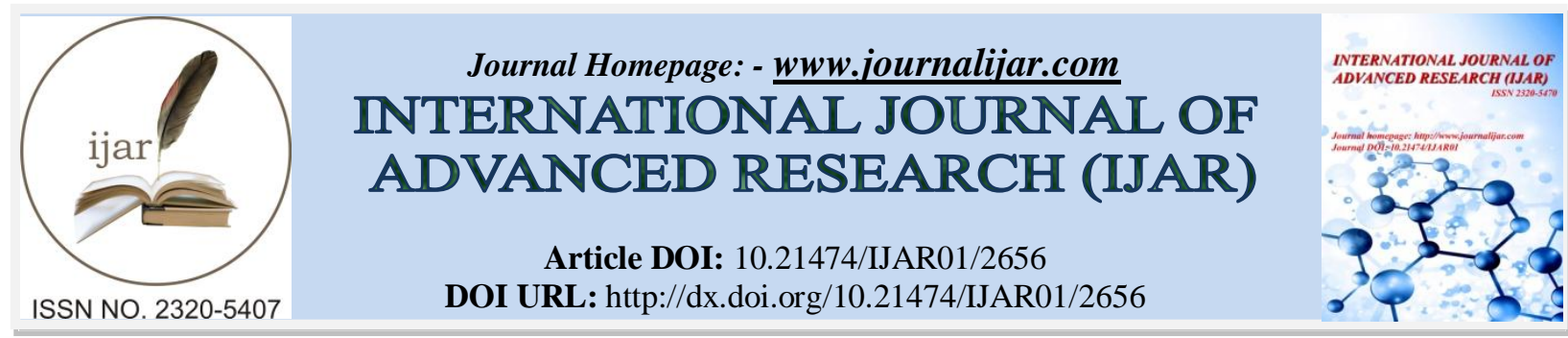

RESEARCH ARTICLE

\title{
PREVALENCE AND PATTERN OF CHEST TRAUMA IN SAUDI ARABIA：A SINGLE-CENTER EXPERIENCE.
}

\author{
Majed al Mourgi ${ }^{1,2}$, Tamer M. Abdelrhman ${ }^{1,3}$, Ahmad Newaf haj Hussein ${ }^{2}$, Khaled Fares Almekdad ${ }^{2}$, Saad \\ Saleh S. Alobaysi ${ }^{4}$, Saad Saleh A Alobaysi ${ }^{4}$, Muhannad A. Alshehri ${ }^{4}$, Mohammed Khalid F. Alharthi ${ }^{4}$, \\ Abdulsalam Saed M. Alghamdi ${ }^{4}$ and Abdullah Mastour A. Alqarni ${ }^{4}$. \\ 1. Department of Surgery, Al Taif University, KSA. \\ 2. Thoracic Surgery, King Abdulaziz Specialist Hospital, Taif, KSA. \\ 3. General Organization for Teaching Hospitals and Institutes, Egypt. \\ 4. MBBS, College of Medicine, Taif University, KSA.
}

\section{Manuscript Info}

\section{Manuscript History}

Received: 30 October 2016

Final Accepted: 29 November 2016

Published: December 2016

Key words:-

Chest injuries; Road traffic accident;

Rib fracture; Saudi Arabia.

\section{Abstract}

Background: Chest injuries are one of the common causes of mortality and morbidity.

Aim: our aim was to analyze the prevalence, pattern, severity, and management of chest injuries and to outline the outcome and the prognosis in our local setting.

Methods: We conducted this retrospective study in King AbdulAziz Specialist Hospital, Taif, (KSA). A total of 226 patients who presented to the ER with chest injuries were included in the study. The data collected and entered into the proforma.

Results: The highest rate of injuries was observed in the age group of 21-30 years old. Most patients were male (87.2\%) and involved in RTAs $(86.7 \%)$. The most common clinical type of chest injury was lung contusion, followed by rib fractures and pneumo-thorax. Head trauma was the most common extra-thoracic injury, followed by injury to the extremities. $75.2 \%$ of patients were managed conservatively, $25.2 \% \%$ required tube thoracostomy, while $1.8 \%$ required thoracotomy. Length of hospital stay was increased in patients with extra-thoracic injuries and those who were intubated. Conclusion: RTAs constitute the leading cause of chest trauma in our locality. Establishing a national chest trauma database is an essential step toward decreasing the burden of traumatic chest injuries.

Copy Right, IJAR, 2016,. All rights reserved.

\section{Introduction:-}

The Kingdom of Saudi Arabia (KSA) is among many developing countries that have suffered a major burden of morbidity and mortality due to injuries. Preventable injuries are the second leading cause of death, accounting for about a fifth of all reported fatalities in the country; however, little is known about their pattern and outcomes [1]. As the population of KSA is relatively young, injuries have significant implications on the country's health and prosperity; $40 \%$ of the population is 19 years old and younger [2]. Road traffic accidents (RTA) and falls are the 
most common causes of trauma, and $21 \%$ of victims are admitted for chest trauma [3]. The high motorization rate in Middle Eastern countries has increased the incidence of RTA-related fatalities [4].

Injuries to the thorax are the third most common injuries in trauma patients, after injuries to the head and extremities. The presence of thoracic injuries in the setting of multi-systemic trauma can significantly increase patient mortality [5], and thoracic injuries are involved in about a quarter of all trauma-related deaths [6]. Thoracic lesions from chest injuries range from simple chest wall contusions to vital organ injury, and they are often associated with other extra-thoracic injuries, particularly to the abdomen and long bones [7].

According to Advanced Trauma Life Support ${ }^{\circledR}$ protocol, the following six injuries must be rapidly diagnosed (the lethal six) as they have the potential to cause death: airway obstructions, tension pneumo-thorax, flail chest, cardiac tamponade, open pneumo-thorax, and massive hemo-thorax. In addition, the following six injuries may be delayed or masked by other injuries (the hidden six): trachea-bronchial disruption, pulmonary contusion, traumatic disruption of the aorta, blunt cardiac injury, esophageal perforation, and diaphragmatic tear [8]. Accurate assessment of a trauma patient at high risk for major potentially life-threatening chest injuries is critical. Moreover, to achieve optimal patient outcome and significantly decrease morbidity and mortality, patients need aggressive management of the chest injuries along with accurate treatment of co-existing injuries [9].

In spite of the high mortality rates, the outcome and prognosis for the majority of patients with chest trauma are excellent. Most cases, even with life-threatening thoracic injuries, may require either non-invasive therapy or, at most, simple intervention such as drainage of the pleural space via tube thoracostomy [5].

Since most chest trauma is preventable, it is important to understand the etiology, injury pattern, and management protocols [10]. However, in KSA, there is a lack of information regarding the magnitude and management of chest injuries. Accordingly, we conducted this retrospective study to analyze the epidemiology, pattern, severity, and management of chest injuries. In addition, we outlined the outcome and the prognosis of this life-threatening condition in our local setting. The results will provide the basis for planning prevention strategies and establishment of treatment protocols.

\section{Materials \& Methods:- \\ Methods:-}

This retrospective study was conducted at King Abdul-Aziz Specialist Hospital (KASH), a tertiary hospital in Taif city with a 500-bed capacity providing many specialties and services. Taif is a city in the Makkah Province of Saudi Arabia at an elevation of $1,879 \mathrm{~m}$ on the slopes of the Sarawat Mountains. It has a population of around 1 million; the city has a pleasant year round climate throughout the year, so it is one of the most popular holiday resorts.

\section{Ethical approval:-}

Ethical approval was obtained from the Research Ethics Committee of KASH. Confidentiality of patients was preserved by not including names or identifying details in the proforma.

\section{Inclusion criteria:-}

All patients were included in the study if they presented to the emergency department with chest injuries and were admitted and treated from November 2013 to March 2016.

\section{Medical Records:-}

A total of 226 patients were included in the study. Data collected and entered into the proforma consisted of demographics, causes and mechanisms of injury, pattern of lesions, presence of associated injuries, management and outcome including length of hospital stay, complications, and mortality. Records were traced manually from the Medical Records department; cases with missing records and those with incomplete data were excluded.

\section{Statistical analysis:-}

Data were entered into a computer and analyzed using SPSS software version 14 with the help of a medical statistician. Categorical variables were summarized as proportions and frequencies for categorical variables and analyzed using the chi-square test. Continuous variables were expressed as mean and standard deviation. Correlation analysis was performed using Spearman's correlation. Significance was defined as a $p$-value of $\leq 0.05$. 


\section{Results:-}

This study included a total of 226 patients with chest injury; the mean age was 32.1 years (range, 1.5-88 years), and the highest rate of injuries (approximately one-third) was observed in those aged 21-30 years; $70 \%$ of the injuries were in those aged $11-40$ years. Regarding sex, $87.2 \%$ of the patients were men $(n=197)$ and $12.8 \%$ were women $(\mathrm{n}=29)$.

The majority of patients $86.7 \%(n=196)$ were involved in RTA; motor vehicle $(n=190)$, Pedestrian $(n=5)$, motorcycle $(\mathrm{n}=1)$. While fall from height was $4.8 \%$, blunt assault was $2.2 \%$, stab $4.8 \%$ and firearm injuries was $1.3 \%$.

The most common injuries were lung contusion $(58.8 \%)$, rib fractures $(48.6 \%),(\mathrm{n}=110)$, and pneumo-thorax (25.2\%; Table 1). Fractures of the first rib were present in $23.6 \%$ of patients. The most common bone fractures other than ribs were those of the thoracic spine (13.7\%) and scapula (10.6\%; Table 1).

Table 1:- Distribution of patients according to diagnosis of chest injury

\begin{tabular}{|c|c|c|}
\hline Injury type & No. of patients & Percent \% \\
\hline Rib fracture & 110 & 48.6 \\
\hline Pneumo-thorax & 57 & 25.2 \\
\hline Hemo-thorax & 31 & 13.7 \\
\hline Hemopneumo-thorax & 37 & 16.4 \\
\hline Lung contusion & 133 & 58.8 \\
\hline Flail chest & 3 & 1.3 \\
\hline Diaphragmatic injury & 5 & 2.2 \\
\hline Pneumo-pericardium & 3 & 1.3 \\
\hline Pneumo-mediastinum & 7 & 3.1 \\
\hline Surgical emphysema & 32 & 14.2 \\
\hline Sternal fracture & 12 & 5.3 \\
\hline Scapular fracture & 24 & 10.6 \\
\hline Clavicular fracture & 14 & 6.2 \\
\hline Thoracic spine fracture & 31 & 13.7 \\
\hline
\end{tabular}

The most common extra-thoracic injuries were those to the head $(35.8 \%)$ and extremities $(25.6 \%)$, while extra thoracic spine injuries were (18.5\%). The abdominal injuries were 36 patients (15.9\%) which of them 15 patients with Splenic injuries, 9 patients with liver injuries, 10 patients with spleen and liver injuries, one patient with spleen and left kidney injuries and one patient with bladder injuries.

Conservative treatment was applied for $75.2 \%$ of the patients, followed by tube thoracostomy (25.2\%), intubation $(23.8 \%)$, tracheostomy (2.6\%), and thoracotomy (1.8\%) (Table 2).

Table 2:- Distribution of patients according to management of chest injury.

\begin{tabular}{|c|c|c|c|c|}
\hline & & & & \multirow{5}{*}{25.2} \\
\hline Conservative / observation & \multicolumn{2}{|c|}{170} & 75.2 & \\
\hline Right tube thoracostomy & 26 & \multirow{3}{*}{57} & 11.5 & \\
\hline Left tube thoracostomy & 25 & & 11 & \\
\hline Bilateral tube thoracostomy & 6 & & 2.6 & \\
\hline Thoracotomy & & & & \\
\hline Intubation & & & & \\
\hline Tracheostomy & & & & \\
\hline
\end{tabular}

For management of extra-thoracic injuries craniotomy was done for tow patients, laparotomy done for 16 patients and orthopedic interventions was done for 53 patients. Co-morbidities on admission were found in 26 patients with hypertension, 23 patients with diabetes, 6 patients with bronchial asthma and two patients with hypothyroidism. Over half of patients had a hospital stay of less than one week, while over $80 \%$ had a stay of less than two weeks. 
Table 3:- Rib fractures and associated lung injuries.

\begin{tabular}{|c|c|c|c|c|c|c|c|c|}
\hline No. of ribs (no. of patients) & \multicolumn{2}{|c|}{ Pneumo-thorax } & \multicolumn{2}{|c|}{ Hemo-thorax } & \multicolumn{2}{|c|}{ Hemopneumo-thorax } & \multicolumn{2}{|c|}{ Contusion } \\
\hline & yes & no & yes & no & yes & no & yes & no \\
\hline 1-2(20) & 8 & 12 & 2 & 18 & 4 & 16 & 8 & 12 \\
\hline 3-5 (37) & 15 & 25 & 5 & 32 & 7 & 30 & 24 & 13 \\
\hline$>5 \quad(53)$ & 10 & 34 & 11 & 42 & 12 & 41 & 34 & 19 \\
\hline$\chi^{2}$ & \multicolumn{2}{|c|}{2.8} & \multicolumn{2}{|c|}{1.5} & \multicolumn{2}{|c|}{0.19} & \multicolumn{2}{|c|}{4.1} \\
\hline $\mathbf{p}$ & \multicolumn{2}{|c|}{0.2} & \multicolumn{2}{|c|}{0.4} & \multicolumn{2}{|c|}{0.9} & \multicolumn{2}{|c|}{0.1} \\
\hline
\end{tabular}

Of the 226 total patients, nine died, for a mortality rate of $4 \%$. Twelve patients $(5.3 \%)$ had complications; two cases with acute respiratory distress, and one case with aspiration pneumonia. The other complications were related to associated extra-thoracic injuries and included two cases of paraplegia, two of deep vein thrombosis, one of pulmonary embolism, one of urinary tract infection, one of arrhythmia, one of anoxic encephalopathy, and one of brain infarction and sepsis.

There was no association between number of rib fractures and type of lung injury (Table 3).

The mortality rate was negatively associated with the Glasgow Coma Scale (GCS) score $(\mathrm{p}=0.0001)$ and positively associated with intubation $(\mathrm{p}=0.0001)$. The length of hospital stay was negatively associated with GCS ( $\mathrm{p}=$ $0.0001)$ and positively associated with the presence of extra-thoracic injuries $(p=0.001)$ and intubation $(p=0.0001$; Table 4).

Table 4:- Factors contributing to length of hospital stay and mortality

\begin{tabular}{|c|c|c|c|c|}
\hline & \multicolumn{2}{|c|}{ Correlation with LOH stay } & \multicolumn{2}{c|}{ Correlation with mortality } \\
\hline Factors & $\mathrm{r}$ & $\mathrm{p}$ & $\mathrm{r}$ & $\mathrm{p}$ \\
\hline Age & 0.076 & 0.255 & -0.066 & 0.321 \\
\hline Sex & 0.002 & 0.975 & -0.078 & 0.242 \\
\hline Rib fracture & 0.062 & 0.355 & -0.008 & 0.910 \\
\hline Extra-thoracic injury & 0.219 & $0.001^{*}$ & 0.061 & 0.361 \\
\hline GCS & -0.452 & $0.0001^{*}$ & -0.447 & $0.0001^{*}$ \\
\hline Intubation & 0.365 & $0.0001^{*}$ & 0.352 & $0.0001^{*}$ \\
\hline Co morbidity & 0.087 & 0.193 & 0.029 & 0.660 \\
\hline
\end{tabular}

r, correlation coefficient; GCS, Glasgow Coma Scale score; * $\mathrm{p}<0.05$

Having a flail chest was positively associated with complications $(\mathrm{p}=0.029)$. In addition, having a flail chest was also positively associated with mortality $(\mathrm{p}=0.009)$, as was the presence of lung contusion $(\mathrm{p}=0.010$; Table 5$)$. Head injury was positively associated with mortality $(\mathrm{p}=0.001$; Table 5$)$.

Table 5:- Effect of chest injuryand extrathoracic injury on patient outcome

\begin{tabular}{|c|c|c|c|c|c|c|}
\hline \multirow{2}{*}{} & \multicolumn{2}{|c|}{ Uneventful } & \multicolumn{2}{c|}{ Complicated } & \multicolumn{2}{c|}{ Mortality } \\
\cline { 2 - 6 } & $\mathrm{r}$ & $\mathrm{p}$ & $\mathrm{r}$ & $\mathrm{p}$ & $\mathrm{r}$ & $\mathrm{p}$ \\
\hline Pneumothorax & 0.103 & 0.124 & -0.092 & .168 & -0.014 & 0.833 \\
\hline Hemothorax & -0.018 & 0.785 & 0.078 & 0.245 & -0.015 & 0.818 \\
\hline Hemopneumothorax & 0.048 & 0.474 & -0.051 & 0.442 & -0.029 & 0.665 \\
\hline Contusion & -0.124 & 0.063 & 0.038 & 0.574 & 0.170 & $0.010^{*}$ \\
\hline Flail Chest & -0.104 & 0.118 & 0.145 & $0.029^{*}$ & 0.174 & $0.009^{*}$ \\
\hline Rib fracture & -0.050 & 0.454 & 0.071 & 0.290 & -0.008 & 0.910 \\
\hline Diaphragmatic injury & 0.046 & 0.495 & -0.036 & 0.594 & -0.031 & 0.647 \\
\hline Head injury & -0.139 & $0.036^{*}$ & 0.029 & 0.667 & 0.225 & $0.001^{*}$ \\
\hline Abdomenal injury & -0.04 & 0.52 & 0.05 & 0.38 & -0.02 & 0.68 \\
\hline Pelvic injury & -0.013 & 0.847 & 0.060 & 0.368 & 0.013 & 0.849 \\
\hline Extremities injury & -0.06 & 0.32 & 0.10 & 0.10 & 0.05 & 0.43 \\
\hline
\end{tabular}

$\mathrm{r}$, correlation coefficient; $* \mathrm{p}<0.05$ 


\section{Discussion:-}

With rapid industrialization of cities, urbanization, and a tremendous increase in high speed traffic flow, trauma in general and chest trauma in particular are increasing. Thus, a requirement of augmented care, trained personnel, and sophisticated equipment for saving the life of trauma patients has increased [11]. In the current study, the highest rate of chest injuries was observed in those aged 21-30 years, and most injuries (87.2\%) were in adult males. These observations are similar to those from previous reports [7, 12-14] suggesting that males and younger individuals likely have more chest injuries because they are more active and mobile, thus increasing the risk of trauma.

RTAs caused $86.7 \%$ of all cases. This is similar to the results of Ayman et al. [15], who reported that RTAs caused $81.25 \%$ of chest injuries in KSA as well as in other countries [7, 16, 17]. However, other studies [12, 18, and 19] reported that penetrating trauma was the main cause of chest injuries. Alabadani et al. [19] attributed the predominance of penetrating trauma to the ownership and use of guns in Yemen.

Contrary to previous findings where comorbidities significantly affected patient outcome [20], in the present study, where $25.2 \%$ had underlying comorbid diseases on admission, including hypertension, diabetes mellitus, and bronchial asthma, comorbidities did not significantly influence mortality.

Lung contusion was the most common (58.8\%) chest injury, which was similar to the findings of Misauno et al. [21]. This was followed by rib fractures (48.6\%) and pneumothorax (25.2\%). Other studies [7, 22, and 23] reported that rib fractures were the most common chest injury.

Pasha et al. [10] found an incidence of rib fractures in $76.2 \%$ of cases with chest injury and reported a significant association between the number of fractured ribs and the presence of lung complications; however, this association was not observed in the current study. Although, the high incidence (48.6\%) of first rib fracture in the current study was surprising, suggesting a potential need to focus on this. Patients with pneumothorax were managed firstly with needle decompression then with tube thoracostomy.

Other thoracic bony injuries, mainly thoracic spine fracture, followed by scapula, clavicle, and sternum fracture, were observed and treated conservatively, although one case of clavicle fracture with excessive displacement was treated with internal fixation and three cases with thoracic spine fracture were treated with spinal fixation. These data are consistent with the results from a retrospective study of blunt chest trauma in Baltimore, Maryland suggesting that severe thoracic parenchymal injury can be present with chest injuries, even in the absence of thoracic bony fractures [24].

The presence of associated extra-thoracic injuries is an important determinant of outcome in chest injury patients as it increases the risk of complications. To reduce mortality and morbidity associated with chest injuries, early recognition and treatment of these injuries is important [5]. In the present study, head trauma was noted as the most common (35.8\%) extra-thoracic injury, which is consistent with some previous studies [7, 25, and 26]. However, other studies have reported musculoskeletal injury to be the most predominant extra-thoracic injury (around 50\%) $[10,27]$, whereas it was the second most common $(25.6 \%)$ in the current study.

Most chest injuries can be conservatively managed with observation, adequate analgesia, monitoring, and bed rest, and this was applied for 170 of the 226 patients $(75.2 \%)$ included in the present study. However, in cases of pneumothorax and hemothorax, tube thoracostomy is indicated for drainage. It is considered generally safe, if conducted properly and sterilely [28]. Fifty-seven (25.2\%) of the patients underwent tube thoracostomy, with no major complications. This is similar to the rate reported by Demirhan et al. [29].

There is a general agreement that thoracotomy is not mandatory in chest injury in the absence of definite indication [7]. Only four (1.8\%) of the current patients required thoracotomy, all due to extensive hemothorax (more than 1500 $\mathrm{ml}$ drained by chest tube). In addition, five cases with diaphragmatic tear underwent repair via laparotomy.

Endotracheal intubation was performed in 54 (23.8\%) patients. In most cases, intubation was performed owing to associated head trauma, with some for lung contusion and one for flail chest. Of those undergoing intubation, eight patients also underwent tracheostomy after 14 days of intubation. The need for endotracheal intubation was associated with mortality, thus this relationship should be further explored. Similarly, Balkan et al. [30] reported that 
endotracheal intubation following blunt chest trauma was associated with poor prognosis, although they reported a higher rate of intubation.

The length of hospital stay is an important measure of morbidity and bed occupancy rate. In the present study, the average hospital stay was 10.5 days (median, 7 days). The length of hospital stay increased in patients with intubation and extrathoracic injuries, primarily head injuries (fractured base of skull, subarachnoid and subdural hemorrhage), as well as some cases with lower limb injuries that required open reduction internal fixation. These findings are consistent with other study [7].

Overall mortality in the current study was 4\%, which is consistent with that reported in other studies [7, 25-27]. Mortality was affected by GCS, intubation, lung contusion, flail chest, and associated head injury. Other study found other predicting factors of mortality such as age, sex, associated comorbidities, associated injuries, number of fractured ribs, admission to the intensive care unit, and the need for artificial ventilation [25].

\section{Conclusion:-}

Chest trauma is imposing an increasingly severe burden on the health infrastructure of the developing world. The current study highlights the patterns of chest trauma and its management in a single center in Taif, KSA. Management of traumatic chest injuries should be a matter of extreme emergency. Establishing a national chest trauma database is an essential step in decreasing the burden of traumatic chest injuries.

\section{Conflict of Interest:-}

Author declares that they have no conflict of interest.

\section{Acknowledgments:-}

The author wishes to acknowledge with thanks Dr. Rehab Ahmed Karam for her help with medical statistics.

\section{References:-}

1. Al-Naami MY, et.al (2010). Trauma care systems in Saudi Arabia: an agenda for action. Ann Saudi Med.; 30(1): 50-8.

2. Alghnam S, et.al (2014). Burden of traumatic injuries in Saudi Arabia: lessons from a major trauma registry in Riyadh, Saudi Arabia, Ann Saudi Med.; 34(4): 291-296.

3. National Trauma Databank 2014 annual report American College of Surgeons, Chicago. www.facs.org/ntdbannualreport.

4. El-Menyar A, et.al (2016). Clinical Presentation and Time-Based Mortality in Patients with Chest Injuries Associated with Road Traffic Accidents, Arch Trauma Res.; 5(1): e31888.

5. Elbaih AH, Elshapowry IM (2016). Evaluation of thoracic trauma severity score in predicting the outcome of isolated blunt chest trauma patients. Int J Surg Med.; 2(3): 100-6.

6. Wison RF, Murray Antonio DR (2001). Non penetrating thoracic injury. Surg Clin North Am.; 57: 17-36.

7. Lema MK, et.al (2011). Pattern and outcome of chest injuries at Bugando, Medical Centre in Northwestern Tanzania. J Cardiothorac Surg.; 6: 7.

8. Tai NRM, Boffard KD (2003). Thoracic trauma: principle of early management. Trauma; 5: 123-136.

9. Griwan MS (2016). To study the etiology, pattern and short term outcome of chest injuries as prospective study. Int J All Res Educ Scientific Meth; 4(3): 6-11.

10. Pasha MA, et.al (2015). A 10-year retrospective review of chest trauma in Hospital Universiti Sains Malaysia. J Dent Med Sci.; 14(8): 68-74.

11. Shah JV, Solanki MI (2015). Analytic Study of Chest Injury.IJSS Journal of Surgery; 1:5-9.

12. Ali N, Gali BM (2004). Pattern and management of chest injuries in Maiduguri, Nigeria. Ann Afric Med.; 3 : 181-184.

13. Adem AA, et.al (2009). Chest injuries in Tikur Anbessa Hospital, Addis Ababa, Ethiopia: 3-year experience. East Central Afric J Surg.; 6: 11-14.

14. Ekpe EE, Eyo C (2014). Determinants of mortality in chest trauma patients, Niger J Surg.; 20(1): 30-34.

15. Ayman G, Mohamed A (2013). Role of emergency vats in blunt chest trauma patients. Br J Sci.; 9(2): 37-42.

16. Kesieme EB, et.al (2011). Profile of thoracic trauma in two semiurban university hospitals in Nigeria. Professional Med J.; 18(3): 373-379. 
17. Saaiq M, Shah SA (2008). Thoracic Trauma: Presentation and Management Outcome. J Coll Physicians Surgeons Pakistan. 18(4): 230-233.

18. Maxwell RA, et.al (2004). Use of presumptive antibiotics following tube thoracostomy for traumatic haemopneumothorax in the prevention of empyema and pneumonia; a multi-center trial; 57(4): 742-8.

19. Albadani MN, Alabsi NA (2011). Management of Chest Injuries: A prospective study Yemeni J Med Sci.; 5: 23-27.

20. Battle CE, et.al (2012). Risk factors that predict mortality in patients with blunt chest trauma: A systematic review and meta-analysis. Injury.; 43: 8-17.

21. Misauno MA, et.al (2007). Severe chest trauma in Jos, Nigeria: Pattern and outcome of management. Nigerian J Orthopaed Trauma.; 16(2): 64-66.

22. Wanek S, Mayberry JC (2004). Blunt thoracic trauma: flail chest, pulmonary contusion, and blast injury. Crit Care Clinic; 20: 71-81.

23. Massaga FA, Mchembe M (2010). The Pattern and Management of Chest Trauma at Muhimbili National. Hospital, Dar el Salaam. East Centr Afric J Surg.; 15: 124-29.

24. Shorr RM, et.al (1987). Blunt thoracic trauma. Ann Surg.; 206(2): 200-205.

25. Kulshrestha P, et.al (2004). Profile of chest trauma in a level I trauma center, J Trauma.; 57: 576-581.

26. Segers P, et.al (2001). Thoracic trauma: an analysis of 187 patients. Acta Chir Belg.; 101: 277-282.

27. Senor BV, et.al (2004). Predictors of outcome in blunt chest trauma. Arch Bronchopneumol; 40: 489-494.

28. Sirmali M, et.al (2003). A comprehensive analysis of traumatic rib fractures, morbidity, mortality and management. Eur J Cardiothoracic Surg.; 24: 133-138.

29. Demirhan R, et.al (2009). Comprehensive analysis of 4205 patients with chest trauma: a 10-year experience. Interact Cardiovasc Thorac Surg.; 9(3): 450-453.

30. Balkan ME (2002). Emergency Thoracotomy for Blunt Thoracic Trauma. Ann Thorac Cardiovasc Surg.; 8(2): 78-82. 